\title{
On the effect of neoclassical flows on intrinsic momentum in ASDEX Upgrade Ohmic L-mode plasmas
}

\author{
W A Hornsby ${ }^{1}$, C Angioni ${ }^{1}$, E Fable ${ }^{1}, \mathbf{P}$ Manas $^{1}, \mathbf{R}$ \\ McDermott $^{1}$, A G Peeters ${ }^{2}$, M. Barnes ${ }^{3}$ and F. Parra ${ }^{3}$ and \\ the ASDEX Upgrade Team \\ ${ }^{1}$ Max-Planck-Institut für Plasmaphysik, Boltzmannstrasse 2, D-85748 \\ 2 Theoretical Physics V, Dept. of Physics, Universität Bayreuth, Bayreuth, \\ Germany, D-95447 \\ ${ }^{3}$, Rudolf Peierls Centre for Theoretical Physics, University of Oxford, 1 Keble \\ Road, Oxford, OX1 3NP, United Kingdom \\ E-mail: william.hornsby@ipp.mpg.de
}

\begin{abstract}
A gyro-kinetic analysis of intrinsic rotation is presented for the ASDEX Upgrade tokamak. The gyro-kinetic turbulence code, GKW and the neoclassical transport code, $\mathrm{NEO}$ are coupled so that the neoclassical equilibrium distribution function is included in the background distribution function in the gyro-kinetic turbulence simulation. This implementation is benchmarked against a similar implementation in the gyro-kinetic code, GS2 (W. Dorland et al. Phys. Rev. Lett (2000)) and against analytical predictions.

A quasi-linear and non-linear gyro-kinetic turbulence analysis is performed on Ohmic L-mode ASDEX Upgrade plasmas showing that the symmetry breaking effects due to neoclassical background flows can produce significant toroidal momentum transport. While its magnitude is of the order of other symmetry breaking mechanisms, such as the Coriolis pinch, up-down asymmetry in the magnetic flux surfaces and $\mathrm{E} \times \mathrm{B}$ flow shear, the flow gradients it can sustain are appreciably smaller than the maximum gradients measured at the mid-radius of the ASDEX Upgrade tokamak core, which can be up to an order of magnitude larger.

It is found that the gradient of the diamagnetic flow, and therefore the second derivatives of the density and temperature gradients are critical to the production of residual toroidal momentum flux. A quasi-linear estimate indicated that the second derivatives required to match the experimental flow gradient are up to an order of magnitude higher than the measured second derivatives. This analysis suggests that turbulent transport driven by neoclassical flows is not sufficient to explain the maximum flow gradients observed in ASDEX Upgrade.
\end{abstract}

\section{Introduction}

It has been observed in multiple tokamak fusion experiments that, even without sources of external momentum, a confined plasma can have a toroidal rotation [1]. This is known as intrinsic rotation and is of particular interest to a reactor plasma where the application of external torque will be comparatively small. Here the toroidal rotation profiles are determined largely by plasma transport processes. 
Large toroidal flow gradients have been measured and this, through the effect of flow shear, can have a stabilising effect on plasma turbulence $[2,3,4]$. Turbulence causes the majority of heat and particle transport in magnetic fusion devices. Furthermore, it is also known that high rotation and rotation shear is stabilising for large scale Magneto-hydrodynamic (MHD) modes such as Neoclassical tearing modes [5] and resistive wall modes [6] and as such an understanding of the sources and transport of toroidal rotation is of widespread interest and importance.

Toroidal momentum transport is closely linked to symmetry breaking in the direction along the strong background magnetic field [7, 8]. At lowest order (in expansion parameter $\rho_{*}=\rho_{i} / R_{0}$ ) and in a non-rotating state the gyro-kinetic equation used to describe turbulent fluctuations, has a series of symmetries that prohibits momentum transport $[9,10]$. Toroidal momentum transport is intrinsically linked to mechanisms that break these symmetries.

The total ion toroidal momentum flux, $\Pi_{i}$, which is related to the Reynolds stress can be written as a sum of the symmetry breaking terms $[9,11]$ in the following form

$$
\Pi_{i}=m_{i} n_{i}\left(\chi_{\phi} u^{\prime}+M_{\|} \gamma_{E}+V_{\phi} u+\Pi_{i, r e s}\right),
$$

when the terms can be ordered small. Where $u=R \Omega / v_{t h i}$ is the toroidal $E \times B$ ion flow Mach number and $u^{\prime}=-\left(R^{2} / v_{t h i}\right) \nabla \Omega$, is the toroidal flow gradient. The first term is the diffusive flux. The second term is the toroidal momentum flux generated by $E \times B$ perpendicular shear [12, 13, 14, 15, 16] flows, with shear rate, $\gamma_{E}[17,18]$. The third is the Coriolis pinch [19, 20,21, 22, 23], which can produce in inward or outward flux. The last term is known as the residual stress and includes terms not proportional to the flow or its gradient. $\chi_{\phi}$ is the toroidal viscosity and and $V_{\phi}$ pinch velocity for $E \times B$ flows which are strong functions of the plasma turbulence.

The residual stress component is particularly important as it is considered the source of intrinsic flow. Mechanisms of residual stress generation include, amongst others, up-down flux surface asymmetry [24, 25, 26], higher order corrections to the parallel derivatives [27] and the influence of background neoclassical and diamagnetic flows [28, 29]. Global effects such as profile shearing [30, 31, 32], variation of the safety factor and magnetic shear profile [33], and nonlinear effects such as turbulence spreading [11] and turbulence intensity gradients [34], are also sources of residual stress.

Neoclassical and diamagnetic flows are a function of the density and temperature gradients, collisionality, geometry and plasma current [35] and are usually neglected at lowest order. When included, they introduce a small correction to the equilibrium Maxwellian usually used in gyro-kinetic simulations and a preferential direction. To lowest order, (i.e neglecting flow effects), temperature and density gradients does not cause the transport of toroidal momentum flux. However, through the generation of neoclassical and diamagnetic flows which, in turn break the symmetry of the gyrokinetic equation, they are able to. This mechanism was first outlined analytically in [28], numerically in [29] and an in depth discussion of their symmetry breaking effects in the gyro-kinetic equation can be found in [36, 37, 38].

The per-species flow, $V_{s}$ on a flux surface, can be decomposed into toroidal and poloidal components $[39,40]$. Through the radial force balance equation, the toroidal component of the per species flow can be written in the form [41],

$$
V_{\zeta, s}=\omega_{d, s} R_{0}=-\frac{1}{B_{\theta}} \frac{\partial \phi}{\partial r}-\frac{B_{0}}{B_{\theta}} v_{d i a, s}+\frac{B_{0}}{B_{\theta}} v_{\theta, s} .
$$


The terms on the right hand side being the $E \times B$ flow from radial gradient of the electrostatic potential, the diamagnetic flow related to the gradient in the pressure and the poloidal flow respectively. The latter is largely determined by neoclassical physics and evolves on the ion-ion collisional timescale.

The toroidal momentum flux due to neoclassical and diamagnetic background flows, $\Pi_{s, \text { neo }}$ can be further split into components proportional to the flow, the flow gradient and a residual, in the form

$$
\Pi_{s, \text { neo }}=m_{s} n_{s} R_{0}\left(V_{\phi, d, s} \omega_{d, s}-\chi_{\phi, d, s} \frac{\partial \omega_{d, s}}{\partial r}+\Pi_{r e s, d}\right) .
$$

Where $\chi_{\phi, d}$ and $V_{\phi, d}$ are the diffusivity and convective pinch velocity and $\Pi_{r e s, d}$; the flux from higher order terms, such as the parellel neoclassical heat-flow [29]. In conditions of zero net momentum, i.e when the flows due to diamagnetic effects $\left(\omega_{d}=v_{d} / R_{0}\right)$ and those due to $E \times B$ flows $(\Omega)$ and their radial gradients are opposite in sign and equal in magnitude, a finite residual momentum flux remains which is, in turn, able to maintain a toroidal flow profile due to the differences in the diamagnetic and $E \times B$ diffusion and pinch coefficients [29].

The rest of this paper concerns the gyro-kinetic analysis of intrinsic rotation in the ASDEX Upgrade (AUG) tokamak, using a rotation profile database of ohmic L-mode shots previously outlined and studied in Ref. [42]. Such a comprehensive and systematic analysis of symmetry breaking mechanisms, over such a wide range of parameters, with quantitative comparisons of their corresponding contributions to the experimentally measured toroidal flows has not been previously performed. This paper will concentrate on those mechanisms that are described by the flux-tube model, leaving those that require a radially global analysis to future work.

The analysis here was performed using a modified form of the flux-tube version of the non-linear gyro-kinetic code, GKW [43]. This modification takes the distribution function as calculated by the Eulerian neoclassical transport code, NEO [44, 45, 46] and uses it to modify the equilibrium distribution function used in the gyro-kinetic calculation of turbulent mode stability. Linear and non-linear gyro-kinetic turbulence simulations are used to calculate the toroidal momentum fluxes. These fluxes, and more specifically the flow gradients that they can support, are compared with the experimentally measured values and their relation to other symmetry breaking mechanisms are discussed.

The paper is structured as follows. In section 2 the implementation of the neoclassical background distribution function is described and benchmarked. In section 3 our implementation is applied to the AUG Ohmic database and a quasilinear analysis of intrinsic rotation is performed. Finally in Section 4 our conclusions are drawn.

\section{GKW-NEO implementation}

The flux-tube version of the non-linear Eulerian gyro-kinetic code GKW [43] is used and is interfaced with the Eulerian neoclassical transport code, NEO whose solution is used to introduce background asymmetries into the turbulent system. GKW utilises the parallel (to the magnetic field) velocity, $v_{\|}$and the magnetic moment $\mu=m v_{\perp}^{2} / 2 B$ as its velocity space coordinates both which are normalised to the ion thermal velocity, $v_{t h i}=\sqrt{2 T_{i} / m_{i}}$, the lengths are normalised to the major radius, $R_{0}$ while the 
magnetic field is normalised to the field on the magnetic axis, $B_{r e f}$. In NEO, the velocity space grid uses energy $\epsilon=m v^{2} / 2$ and pitch angle, $\cos \xi=v_{\|} / v$, as the velocity space coordinates and length scales are normalised to the minor radius, $a$. Some care must be taken when converting the solution from NEO into one that can be used by GKW because of this difference.

The gyro-center distribution function, $f_{t o t}$ is decomposed into a slowly evolving equilibrium, $F_{0}$, and small fluctuating component, $\delta f$. The perturbed component is smaller than the equilibrium by a factor of $\rho_{*}=\rho_{i} / R_{0}$, the normalised ion-gyroradius. The plasma is sufficiently collisional so that the background distribution function can furthermore be written as a small deviation from a Maxwellian, $F_{0}=$ $F_{M}+f_{\text {neo. }}$. Similarly the electrostatic potential, $\phi$, can be decomposed into a equilibrium component and a turbulent component, $\phi=\phi_{\text {neo }}+\delta \phi$.

We start with the gyro-kinetic equation in $\left(\mathbf{X}, v_{\|}, \mu\right)$ coordinates, to lowest order in the $\rho_{*}$ expansion and in the electrostatic $(\beta=0)$ limit, given by,

$$
\frac{\partial f_{\text {tot }}}{\partial t}+\frac{\mathrm{d} \mathbf{X}}{\mathrm{d} t} \cdot \nabla f_{\text {tot }}+\frac{\mathrm{d} v_{\|}}{\mathrm{d} t} \frac{\partial f_{\text {tot }}}{\partial v_{\|}}=0 .
$$

In a rigidly rotating frame of reference and ignoring centrifugal effects [47], the equations for the evolution of the gyro-centre, $\mathbf{X}$, and its velocities in the low beta approximation are given respectively by,

$$
\begin{aligned}
& \frac{\mathrm{d} \mathbf{X}}{\mathrm{d} t}=v_{\|} \mathbf{b}+\mathbf{v}_{D}+\mathbf{v}_{E} \\
& m v_{\|} \frac{\mathrm{d} v_{\|}}{\mathrm{d} t}=-\frac{\mathrm{d} \mathbf{X}}{\mathrm{d} t} \cdot[Z e \nabla\langle\phi\rangle+\mu \nabla B] \\
& \frac{\mathrm{d} \mu}{\mathrm{d} t}=0 .
\end{aligned}
$$

The angled brackets, $\langle\cdot \cdot\rangle$, denote the gyro-average operator, defined as, $\langle\phi\rangle=$ $(1 / 2 \pi) \int d \alpha \phi(R+\rho)$, where $\alpha$ is the gyro-phase and $\rho$ is the species gyro-radius, $\vec{\rho}=\rho\left(\vec{e}_{1} \cos \alpha+\vec{e}_{2} \sin \alpha\right)$. The vectors $\vec{e}_{1}$ and $\vec{e}_{2}$ are orthogonal unity vectors perpendicular to the magnetic field. Field aligned Hamada coordinates $(\psi, \zeta, s)$ are used [48] where the contra-variant components of the magnetic field are flux functions and $B^{\psi}=B^{\zeta}=0$. The radial coordinate, $\psi=r / R_{0}$, is a flux label, $r=1 / 2\left(R_{\max }-R_{\min }\right)$ is the minor radius and $R_{\min }$ and $R_{\max }$ respectively are the minimum and maximum major radii.

The drift velocities of the gyro-centres are defined as:

$$
\begin{aligned}
& \mathbf{v}_{D}=\frac{1}{Z e}\left[\frac{m v_{\|}^{2}}{B}+\mu\right] \frac{\mathbf{B} \times \nabla B}{B^{2}}+\frac{2 m v_{\|}}{Z e B} \boldsymbol{\Omega}_{\perp} \\
& \mathbf{v}_{E}=\frac{\hat{b} \times \nabla\langle\phi\rangle}{B}
\end{aligned}
$$

The first being the combined curvature and magnetic gradient drift and the Coriolis drift, while the second is the $E \times B$ drift. $\boldsymbol{\Omega}_{\perp}$ is the angular (toroidal) rotation vector perpendicular to the field, i.e. $\boldsymbol{\Omega}_{\perp}=\boldsymbol{\Omega}-(\boldsymbol{\Omega} \cdot \mathbf{b}) \mathbf{b}$. The form of the gyro-kinetic Vlasov equation, with a general background distribution function, $F_{0}$ is,

$$
\begin{aligned}
& \frac{\partial f}{\partial t}+\left(v_{\|} \mathbf{b}+\mathbf{v}_{D}+\mathbf{v}_{E}\right) \cdot \nabla f-\frac{\mathbf{b}}{m} \cdot(\mu \nabla B) \frac{\partial f}{\partial v_{\|}} \\
& =S\left(F_{0}\right)+C(f) .
\end{aligned}
$$


Here $S\left(F_{0}\right)$ is the source term which includes all terms related to the background distribution function, and has the general form,

$$
\begin{aligned}
& S\left(F_{0}\right)=-\left(\mathbf{v}_{D}+\mathbf{v}_{E}\right) \cdot \nabla F_{0}+\frac{Z e}{m v_{\|}}\left(v_{\|} \mathbf{b}+\mathbf{v}_{D}\right) \cdot \nabla \phi \frac{\partial F_{0}}{\partial v_{\|}} \\
& +\frac{\left(v_{\| \mid} \mathbf{b}+\mathbf{v}_{D}+\mathbf{v}_{E}\right)}{m v_{\|}} \cdot \mu \nabla B \frac{\partial F_{0}}{\partial v_{\|}}
\end{aligned}
$$

The electrostatic potential, and the species are coupled by the gyro-kinetic Poisson equation. Implemented in GKW is a linearised Landau collision operator, $C(f)$, including both pitch-angle and energy-scattering terms [49]. Parallel momentum and energy conservation terms are also implemented. The ion-ion collision frequency is defined as:

$$
\nu_{i i^{\prime}}=\frac{Z_{i}^{2} Z_{i^{\prime}}^{2} e^{4} \ln \Lambda_{i i^{\prime}} n_{i^{\prime}}}{4 \pi \epsilon_{0}^{2} m_{i}^{1 / 2} T_{i}^{3 / 2}}
$$

which is normalised to the trapping/de-trapping rate, $\nu_{*}=q R_{0} \nu_{i i^{\prime}} /\left(v_{t h i} \epsilon^{3 / 2}\right)$. $\ln \Lambda_{i i^{\prime}}$ is the Coulomb logarithm for scattering species $i^{\prime}$ and scattered species, $i, Z_{i}$ is the relative charge.

The neoclassical component of the background distribution function, $f_{\text {neo }}=$ $f_{\text {neo }}\left(s, \psi, v_{\|}, \mu\right)$ and neoclassical electrostatic potential, $\phi_{\text {neo }}=\phi_{\text {neo }}(s, \psi)$ are determined by solution of the drift kinetic equation,

$$
\mathbf{v}_{\mathbf{D}} \cdot \nabla F_{0}+v_{\|} \mathbf{b} \cdot \nabla\left(f_{\text {neo }}+\frac{Z e \phi_{\text {neo }} F_{0}}{T}\right)=C\left(f_{\text {neo }}\right) .
$$

These equations determine the form of the perturbation to the background distribution function and neoclassical potential and are solved for by NEO. These are output, and then in turn read into GKW and added to the background distribution function. The evolution of neoclassical flows occurs on a significantly longer time-scale than the turbulence and therefore can be assumed to be in equilibrium and not time evolving. As such, the influence of turbulence on the neoclasscial equilibrium is neglected here. In the next two subsections, described explicitly are the changes to the equations solved in GKW due to these small perturbations to the background distribution function and electrostatic potential. For brevity the inertial terms are suppressed, however they do not change the result.

\subsection{Corrections to the left hand side}

Firstly, considering the terms related to perturbed distribution function and its derivatives, the left hand side of Eq. 10 has two corrections. First consider the term, $\vec{v}_{E} \cdot \nabla f$, which is non-linear when the $E \times B$ velocity is calculated for the turbulent potential, $\delta \phi$. This term is linear with respect to the neoclassical part of the electrostatic potential, $\phi_{\text {neo }}$, which has a radial and parallel derivative. Therefore this term and its correction have the form,

$$
\begin{aligned}
& \vec{v}_{E} \cdot \nabla f=\frac{\mathbf{b} \times \nabla \phi}{B} \cdot \nabla f+\frac{\mathbf{b} \times \nabla \psi}{B} \frac{\partial \phi_{\text {neo }}}{\partial \psi} \cdot \nabla \zeta \imath k_{\zeta} f+ \\
& \frac{\mathbf{b} \times \nabla s}{B} \frac{\partial \phi_{\text {neo }}}{\partial s} \cdot \nabla \zeta \imath k_{\zeta} f+\frac{\mathbf{b} \times \nabla s}{B} \frac{\partial \phi_{\text {neo }}}{\partial s} \cdot \nabla \psi \imath k_{\psi} f .
\end{aligned}
$$


Gyrokinetic investigation of intrinsic momentum in ASDEX Upgrade L-mode plasmas6

Where $k_{\zeta}$ and $k_{\psi}$ are the binormal and radial wave-numbers respectively. These are the two directions in the code treated pseudo-spectrally. Secondly, the potential modifies the trapping term, giving,

$$
\begin{aligned}
& -\frac{1}{m v_{\|}}\left(v_{\|} \mathbf{b} \cdot\left(\mu \nabla B+Z e \nabla \phi_{\text {neo }}\right)\right) \frac{\partial f}{\partial v_{\|}} \\
& =-\left(\mu \mathcal{F} \frac{\partial B}{\partial s}+\frac{Z}{2 T} \mathcal{F} \frac{\partial \phi_{\text {neo }}}{\partial s}\right) \frac{\partial f}{\partial v_{\|}} .
\end{aligned}
$$

$\mathcal{F}$ is defined as $\vec{B} \cdot \nabla s / B$. The normalisations used and the form of the geometric tensors can be found in Ref. [43].

\subsection{Complete right hand side.}

The background distribution function is split into two stationary components, $F_{0}=$ $F_{M}+F_{\text {neo }}$, the first term is a lowest order Maxwellian and the second is its neoclassical correction. The Maxwellian distribution function and its derivatives have the form:

$$
\begin{aligned}
& F_{M}=\frac{n_{0}}{\pi^{3 / 2} v_{t h i}^{3}} \exp \left(-\frac{\left(v_{\|}-R B_{t} \omega_{\phi} / B\right)^{2}+2 \mu B / m}{v_{t h i}^{2}}\right) \\
& \nabla F_{M}=\nabla P F_{M}-\frac{\mu \nabla B}{T} F_{M} \\
& \partial F_{M} / \partial v_{\|}=-\frac{m v_{\|}}{T} F_{M} \\
& \nabla P=\frac{\nabla n}{n}+\left(\frac{v_{\|}^{2}}{v_{t h}^{2}}+\frac{\mu B}{T}-\frac{3}{2}\right) \frac{\nabla T}{T}+\frac{m v_{\|}}{T} \frac{R B_{t}}{B} \nabla \omega_{\phi}
\end{aligned}
$$

where the last equation is the Maxwellian derivative considering only the thermodynamic quantities, the gradient in the magnetic field we leave separate. Derivatives of the perturbed background are taken numerically using fourth order central differencing. The background Maxwellian has only a radial derivative and $\omega_{\phi}$ is the angular toroidal rotation frequency.

The source term for the Maxwellian component of the background for each species, $s,\left(S\left(F_{0}\right)=S\left(F_{M, s}\right)+S\left(F_{\text {neo,s }}\right)\right)$ is given by:

$$
\begin{aligned}
& S\left(F_{M, s}\right)=-\frac{1}{Z_{s} e B}\left(\frac{m v_{\|}^{2}}{B}+\mu\right)\left(\frac{\mathbf{b} \times \nabla B}{B}\right) \cdot F_{M, s} \nabla P \\
& -\left(\frac{\mathbf{b} \times \nabla \delta \phi}{B}\right) \cdot F_{M, s} \nabla P-\frac{v_{\|} Z e}{T_{s}} \mathbf{b} \cdot \nabla \delta \phi F_{M, s} \\
& -\frac{1}{T}\left(\frac{m v_{\|}^{2}}{B}+\mu\right) \mathbf{b} \cdot \frac{\nabla B \times \nabla \delta \phi}{B} F_{M, s} .
\end{aligned}
$$

Terms involving gradients of the magnetic field, $\mu \nabla B$ cancel out, leaving only terms involving $\nabla P$. In contrast, for the neoclassical correction this cancellation does not occur, the source term has the form, 
Gyrokinetic investigation of intrinsic momentum in ASDEX Upgrade L-mode plasmas7

$$
\begin{aligned}
& S\left(F_{\text {neo }}, s\right)=-\frac{1}{Z_{s} e B}\left(\frac{m v_{\|}^{2}}{B}+\mu\right)\left(\frac{\mathbf{b} \times \nabla B}{B}\right) \cdot \nabla F_{\text {neo, } \mathrm{s}} \\
& -\left(\frac{\mathbf{b} \times \nabla \delta \phi}{B}\right) \cdot \nabla F_{\text {neo }, \mathrm{s}}+\frac{Z_{s} e}{m} \mathbf{b} \cdot \nabla \delta \phi \frac{\partial F_{\text {neo, }}}{\partial v_{\|}}+ \\
& \frac{1}{m v_{\|}}\left(\frac{m v_{\|}^{2}}{B}\right) \mathbf{b} \cdot \frac{\nabla B \times \nabla \delta \phi}{B} \frac{\partial F_{\text {neo, }, \mathrm{s}}}{\partial v_{\|}} .
\end{aligned}
$$

The full form of the normalised source term can be seen in Appendix 1. It should be noted that the first term in Eq. 17 and Eq. 18 are not normally included in gyro-kinetic calculations and are not considered here.

The neoclassical component of the background distribution function, $F_{\text {neo }}$ is calculated and output by NEO, which is then read into GKW and transformed from a harmonic expansion in velocity space into real velocity space as utilised by GKW. In NEO the non-adiabatic part of the distribution function is represented by.

$$
\begin{aligned}
& G_{\text {neo }, \mathrm{s}}= \\
& F_{M, s} \sum_{l=0}^{N_{\xi}} \sum_{m=0}^{N_{E}} \hat{g}_{l, m} P_{l}(\xi) L_{m}^{k(l)+1 / 2}\left(v_{s}^{2} / v_{t h}^{2}\right)\left(v_{s} / v_{t h}\right)^{k(l)},
\end{aligned}
$$

where $P_{l}(\xi)$ are Legendre polynomials and $L_{m}^{\alpha}(v)$ are Laguerre polynomals. $k(l)$ is a mode dependent integer which determines the form of the Laguerre expansion. $\hat{g}_{l, m}$ are the amplitudes and is related to the distribution function, $F_{\text {neo,s }}$ by, $F_{\text {neo,s }}=$ $G_{\text {neo,s }}-F_{M s} \phi_{\text {neo }} e / T_{s}$. As a benchmark of this process, Figure 1 shows the flux surface averaged parallel flow, $\left\langle u_{\|} B\right\rangle=\int d s B \int B d v_{\|} d \mu v_{\| \mid} f$, and critically, its radial gradient as calculated by NEO and by GKW after the transformation is performed for the parameters as described in the next section. It can be seen that agreement is excellent, with the values of the flow matching to within $2 \%$ error.
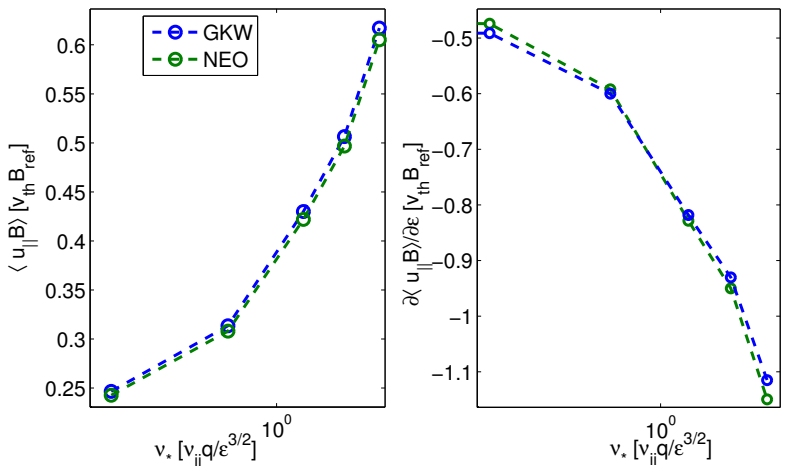

Figure 1. The first order parallel flow (left), $\left\langle u_{\|} B\right\rangle$ and its radial gradient (right) as calculated by NEO (green) and then from the neoclassical distribution function once it has been read and transformed into GKW coordinates (blue) as a function of collisionality. Agreement in flow magnitude is to within $2 \%$. 
Gyrokinetic investigation of intrinsic momentum in ASDEX Upgrade L-mode plasmas8

\subsection{Benchmark}

In this section a benchmark of the independent implementations of the neoclassical background in GKW and GS2 [50] is outlined. The parameters used are based on those described by the Cyclone Base Case [51]: A circular flux surface equilibrium [52], the safety factor, $q=1.4$, magnetic shear, $\hat{s}=0.8$, inverse aspect ratio of the $r / a=0.5$ flux surface, $\epsilon=0.18, R / a=2.78$. The logarithmic density and temperature gradients for both the bulk ions and kinetic electrons are $R / L_{T i}=R / L_{T e}=6.9$, where $R / L_{T i}=R \partial \ln T_{i} / \partial r$ and $R / L_{n i}=R / L_{n e}=2.2$. The ratio of the temperatures is, $T_{e} / T_{i}=1.0$. The value of the normalised gyro-radius (in the case of GKW normalised to the major radius, $\mathrm{R}$ ) is $\rho_{*}=\rho_{i} / R=0.01$.

In GKW the calculation of the radial derivatives requires five equally spaced flux surface calculations for fourth order radial derivatives to be performed. Here, these are chosen to be, $r / a=\left[\begin{array}{lllll}0.49 & 0.495 & 0.5 & 0.505 & 0.51\end{array}\right]$. The radial derivative is only evalauated for the central point with is the local surface of interest.

The grid resolutions for simulations performed with NEO were, $N_{E}=10$, $N_{\xi}=19, N_{\theta}=41$ for the energy, angular polynomials and in the poloidal directions respectively. The Full Fokker-Plank collision operator was used. In GKW, $N_{v_{\|}}=64$, $N_{\mu}=16, N_{s}=30$ in the parallel velocity, magnetic moment and parallel coordinate directions respectively, $N_{x}=21$ radial modes were used. The local flux-tube model is used [53] which is periodic in both the radial and binormal directions and shearperiodic in the parallel direction.

The background toroidal rotation frequency $\left(\omega_{d}=v_{d i a} / R_{0}\right)$ is given by the expression [29],

$$
\begin{aligned}
& \frac{\omega_{\zeta, d} v_{t h i}}{R_{0}}= \\
& \sum_{s}\left\{m_{s} R\left(v_{\|} \frac{\vec{B} \cdot \nabla \zeta}{B}\right) F_{\text {neo }}\right\} / \sum_{s} m_{s} n_{s}\left\{R^{2}\right\},
\end{aligned}
$$

which incorporates the neoclassical and diamagnetic flow components to the total toroidal flow. Here the curly brackets denote the flux surface average. The fluxsurface averaged, gyro-centre fluxes of the toroidal component of the momentum and heat are defined respectively as:

$$
\begin{aligned}
\Pi_{i} & =\Pi_{i}^{\psi}=\left\{\int \mathrm{d}^{3} \mathbf{v} \frac{s_{B} R B_{t}}{B} m v_{\|} \tilde{\mathbf{v}}_{E} \cdot \nabla \psi f\right\} \\
Q_{i} & =Q_{i}^{\psi}=\left\{\int \mathrm{d}^{3} \mathbf{v} \frac{v^{2}}{2} \tilde{\mathbf{v}}_{E} \cdot \nabla \psi f\right\}
\end{aligned}
$$

$\nabla \psi$ is the gradient in the normalised radial coordinate, $\psi=r / R_{0}$. The radial heat flux is written in the form, $Q_{i}=-n_{i} \chi_{i} \nabla T$ where $\chi_{i}$ is the turbulent heat diffusivity.

Figure 2 (bottom) shows the diamagnetic flow and its gradients as a function of the normalised collision frequency for both GS2-NEO and GKW-NEO for the above parameters, showing good agreement between the two codes. Figure 2 shows the turbulent toroidal momentum flux $\left(\Pi_{i}\right)$ normalised to the radial heat flux $\left(Q_{i}\right)$ for a scan in the collisionality as calculated by GKW-NEO (Red) and GS2-NEO (Blue) for a series of linear simulations at the single toroidal wave number of $k_{\zeta} \rho_{i}=0.4242$.

The two implementations show good qualitative agreement and reasonable quantitative agreement. Both codes plateau at low collisionality, although at different 
Gyrokinetic investigation of intrinsic momentum in ASDEX Upgrade L-mode plasmas9
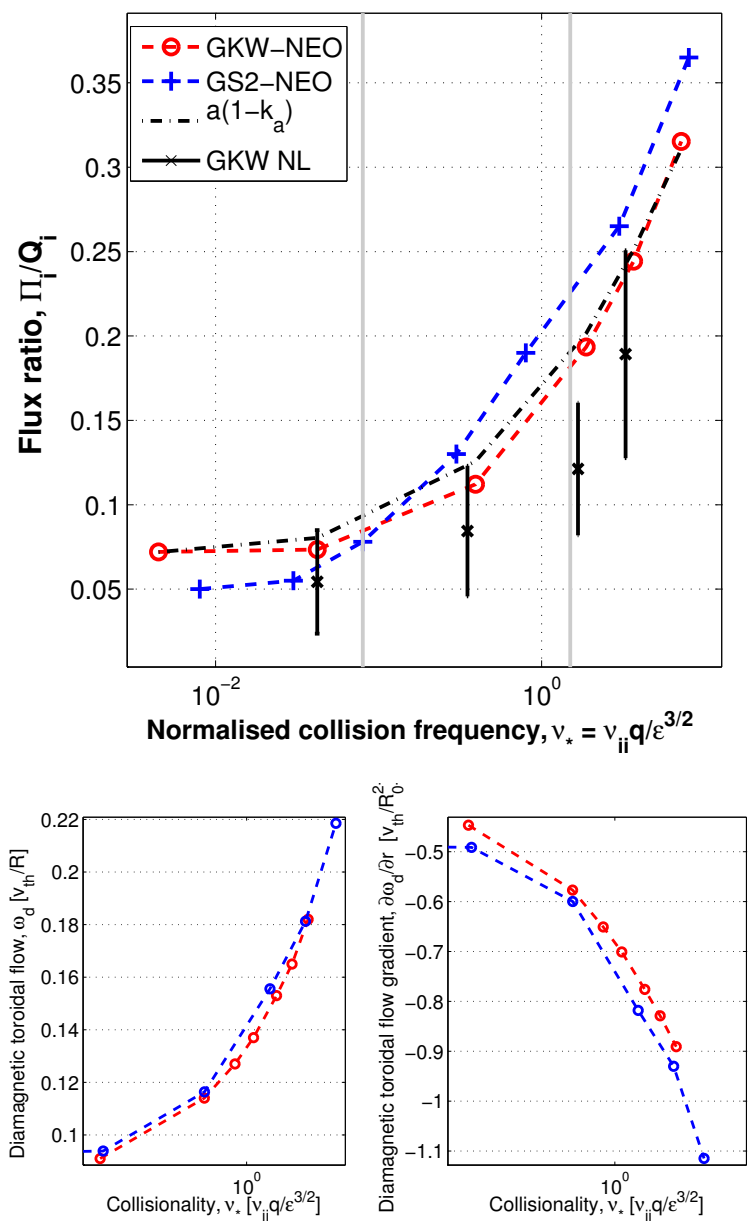

Figure 2. (top) The ratio of the quasi-linear radial flux of toroidal momentum $\left(\Pi_{i}\right)$ with the radial heat flux $\left(Q_{i}\right)$ as a function of collisionality for both GKWNEO (red) and GS2-NEO (blue) at $\rho_{*}=0.089$. Also plotted (black dashed line) is $a\left(1-k_{a}\right)$ where a is fitted to the value from GKW-NEO at the zero collisionality limit. Black crosses represent data from non-linear simulations, the error bars represent the standard deviation of the fluctuations over the statistical steady state part of the simulation. Vertical (grey) lines represent the range of collisionality relevant for AUG L-mode plasmas. (bottom) The toroidal diamagnetic frequency, $\omega_{\zeta, d}$ (left) and its radial gradient, $\partial \omega_{\zeta, d} / \partial r$ (right) for runs with GKW-NEO (blue) and GS2-NEO (red) as a function of the normalised collisionality, GS2-NEO data taken from [29] for $\rho_{*}=0.01$

values, in the banana regime as the background plasma flow is no longer a function of the collision frequency and then increase the momentum flux with collisionality as the background neoclassical flow and flow gradients increase.

Considering the differences in the implementation between the two codes, such as the different form of velocity space grid discretisation, code version and way of taking the radial derivatives, the agreement is satisfactory. It should also be noted that the current implementation of GS2-NEO utilises a version of NEO [44] that expands the 
velocity space in a different set of polynomials to the one coupled to GKW [45] .

Following a similar fluid model analysis to [21] and assuming that the background neoclassical distribution function has the analytical form,

$$
F_{\text {neo }}=F_{M i} \rho_{*} \frac{B_{t}}{B_{p}} \frac{v_{\|}}{v_{t h i}}\left(\frac{R_{0}}{L_{T}}\left(1-K_{1}\right)+\frac{R_{0}}{L_{n}}+\frac{e R_{0}}{T_{i}} \frac{\partial \phi_{\text {neo }}}{\partial r}\right),
$$

it can be shown that the momentum flux generated is directly proportional to $\Pi_{i} / Q_{i} \propto\left(1-K_{1}\right)$ [54], where $K_{1}$ is the dimensionless background flow coefficient [55] which quantifies the amplitude of the collisionality dependent component of the parallel flow and varies between $-0.34<K_{1}<0.69$ for the parameters used here. Inclusion of higher order velocity moments also drive turbulent radial fluxes of momentum which are proportional to $\left(1-K_{1}\right)$. This relation is also plotted in Fig. 2 (black dot-dashed line) for reference with the curves rescaled to match at the zero collisionality limit. Here we demonstrated excellent agreement between this and the values calculated by GKW-NEO.

Furthermore, plotted in Fig. 2 is the flux ratio from nonlinear turbulence simulations for a selection of collisionalities. Non-linear simulations utilised 21 equally spaced $\left(\Delta k_{\theta} \rho_{i}=0.067\right)$ binormal modes and 101 radial modes. Plotted is the mean flux ratio from the statistically steady state phase of the simulation, with the error bar representing the standard deviation in the fluctuation amplitude. It can be seen in general that the nonlinear flux ratio is smaller than the quasi-linearly calculated value. However, for the analysis in this paper a quasi-linear value only slightly overestimates the non-linear values over the collisionalities of interest $\left(\nu_{i *} \leq 1\right)$ and avoids running computationally expensive non-linear turbulence simulations for every database point. This slight overestimation is appropriate to test whether this mechanism of momentum transport production generates large enough fluxes to match the experiment.

\subsection{Influence of flow gradients.}

The momentum flux due to neoclassical background flows is a function of the flow amplitude, its radial gradient and a residual component due to higher order moments [29]. The radial gradient has been shown to provide the largest component [38]. It is possible to isolate the effects of flow gradient itself by modification of the input density and temperature profiles to have the same first derivative (which determines the flow amplitude) but a varying second derivative at the flux surface of interest $(\epsilon=0.18)$ [40]. In this way only the flow gradient changes at the surface of interest. Examples of the ion temperature profiles used can be seen in inlay in Figure 3. This causes a variation the radial gradient of the diamagnetic flow for a fixed value of the flow amplitude at that surface.

Figure 3 shows the quasilinear flux ratio as a function of the diamagnetic flow gradient. This was varied by changing the second derivative of the density and temperature gradients at the flux-surface of interest while keeping a constant first derivative. The variation of the second derivative is parametrised by the dimensionless parameters, $\alpha_{n}=1 /\left(R / L_{n}\right)^{2} R_{0}^{2} \partial^{2} n / \partial r^{2}$ and $\alpha_{T}=1 /\left(R / L_{T}\right)^{2} R_{0}^{2} \partial^{2} T / \partial r^{2}$ where $R / L_{n}$ and $R / L_{T}$ are the logarithmic density and temperature gradients respectively. Plotted in Fig. 3 is the variation in momentum flux while varying independently the density and temperature second derivatives between $\alpha_{n}, \alpha_{T}=[-2,-1,0,1,2]$ for Cyclone base case parameters and for $\nu_{i *}=0.04$. 


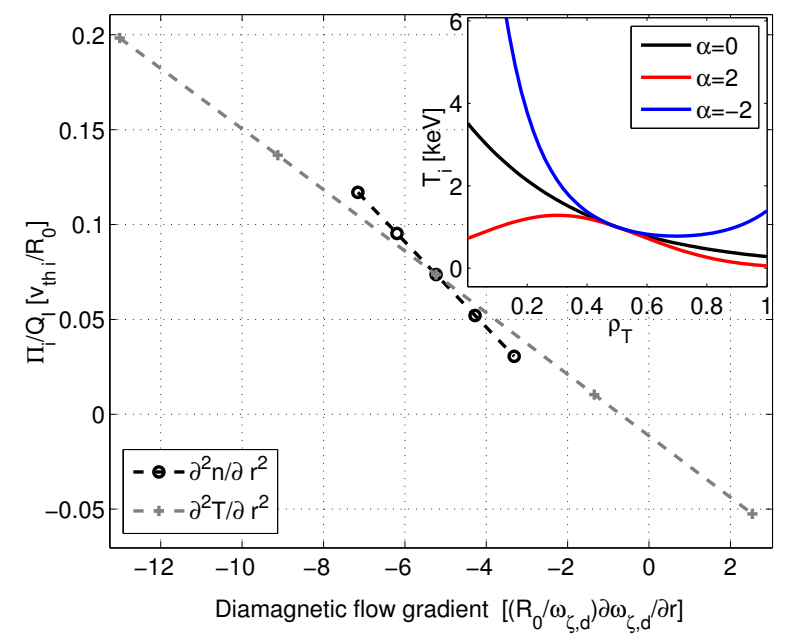

Figure 3. The ratio of the quasi-linear radial flux of toroidal momentum $\left(\Pi_{i}\right)$ with the radial heat flux $\left(Q_{i}\right)$ as a function of the diamagnetic flow gradient which was varied by modification of the second derivatives of the density (black) and temperature (grey) profiles for $\nu_{i *}=0.042$. In inlay are examples of the temperature profiles used.

It is evident that this has a significant effect on the generation of momentum flux. Variations of the second derivatives vary the flow gradient, which in turn causes variation of the momentum flux. We see that the quasi-linear flux ratio is linear in both the second derivative of the density and the temperature. With a small variation of the local gradients it is possible to double the momentum flux, or even cause a complete cancellation of momentum flux generation at finite flow. It has been shown, through non-linear simulations that this effect is reduced when considering contributions from $E \times B$ rotation, as the flux generated by this partially cancels that produced by the neoclassical effect to a point where the momentum flux no longer changes sign [29]. It should be noted that the momentum fluxes in Figures 2 and 3 are calculated in the absence of $E \times B$ flow gradient $\left(u^{\prime}\right)$ and concentrate on the flux generated by the neoclassical flow terms.

\section{Core intrinsic rotation on AUG}

On the spherical tokamak, MAST [56] and conventional tokamak, JET [57], symmetry breaking by neoclassical background flows has been shown to follow qualitative trends and, in some cases, to produce enough turbulent momentum transport to describe the intrinsic rotation profiles and flow reversals seen.

Here the model outlined and benchmarked in the previous sections is applied on the AUG Ohmic intrinsic momentum database which consists of approximately 190 profiles from 22 separate discharges. Large variation of densities (from 1 to $8 \times 10^{19} \mathrm{~m}^{-3}$ ), currents vary from 0.5 to $1 \mathrm{MA}$, edge safety factors, $\mathrm{q}$, from 4.0 to 8.5. All of the plasmas have a lower single null configuration. The major radii were on average $1.63 \pm 0.01 \mathrm{~m}$ and the minor radii were $0.52 \pm 0.02 \mathrm{~m}$. The database and the diagnostics used are described in [42] 

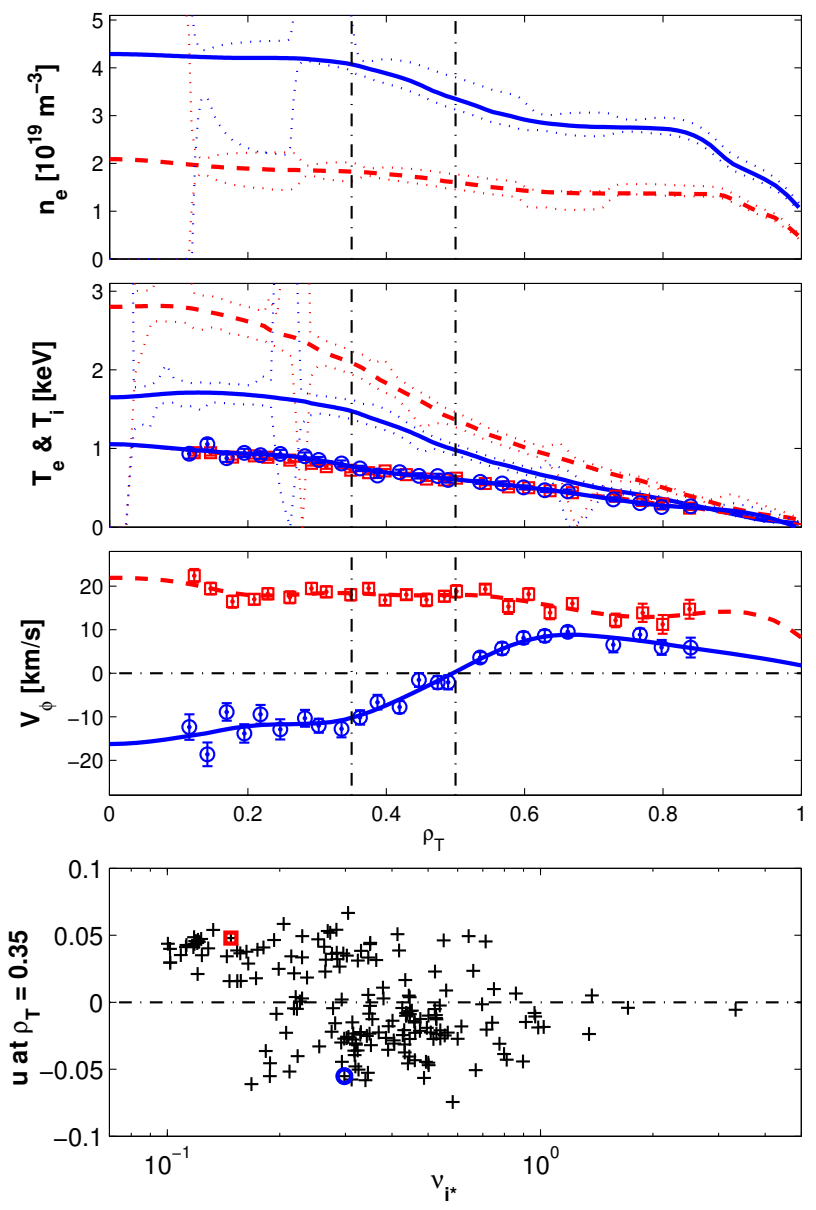

Figure 4. The radial profiles of (top) electron density (upper middle) ion and electron temperature and (lower middle) toroidal flow profile $(u)$ for two shots in the Ohmic database. Red lines are from $t=2.1 \mathrm{~s}$ from shot number 27000 and blue lines from $t=3.8 \mathrm{~s}$ from the same shot. Vertical dashed lines denote the radial positions of the quasi-linear analysis. Dotted lines represent the experimental error in the measurements. (bottom) The toroidal flow Mach number at $\rho_{T}=0.35$ for the whole database, the two points considered above are highlighted.

Plotted in Figure 4 are the profiles of the electron density, temperatures and rotation gradient for two indicative points of the database. The analysis here will concentrate at the radial point (in toroidal flux co-ordinates) $\rho_{T}=0.35$ and a supplementary analysis at $\rho_{T}=0.5$ whose locations are indicated by the vertical dotdashed lines in the figure. These radial positions were chosen as they are within the region where the largest changes in rotation gradient are observed $\left(0.3<\rho_{T}<0.5\right)$ and the position where previous studies were performed $[58,59]$ and so a direct comparison with previous analyses can be made. Locations deeper into the core $\left(\rho_{T}<0.3\right)$ were not considered so as to avoid the effect of sawteeth on our interpretation of the results, while significantly less variation of the flow and its gradient is observed further out $\left(\rho_{T}>0.6\right)$. Plotted in the bottom panel of Fig. 4 are the flow velocities for the 
$\rho_{T}=0.35$ position for every shot in the database as a function of the normalised collision frequency. It can be seen that both positive and negative toroidal flows are observed. The majority of shots have hollow rotation profiles, reflected by negative values of $u^{\prime}<0$ at both radial points $\left(\rho_{T}=0.35\right.$ and $\left.\rho_{T}=0.5\right)$ considered in this paper.

In an attempt to explain the observed flow gradients, the following process was followed. For each case the measured local data at $\rho_{T}=0.35$ (and $\rho_{T}=0.5$ ) were used as inputs for the codes. The magnetic equilibrium is also included through the interface with the MHD equilibrium code, CHEASE [60]. First, the neoclassical flows are calcuated by NEO, then $F_{n e o}$ is read into GKW and incorporated into the background distribution function. Then, in turn, the dominant unstable turbulent mode and the quasi-linear turbulent fluxes at the bi-normal wave number $k_{\theta} \rho_{i}=0.4242$ are calculated in the local, electrostatic limit.

Three kinetic species, deuterium, electrons and boron are considered, with the boron density determined by the value of $Z_{\text {eff }}$ as measured. $Z_{\text {eff }}$ is seen to vary between 1.1 and 2.6. The temperature ratio, $T_{e} / T_{i}$ is seen to vary between $1.36<T_{e} / T_{i}<4.60$. For each database point, and at each radial location, five individual quasi-linear calculations are performed

- With neoclassical background corrections but without $E \times B$ flow effects to calculate the neoclassical residual flux.

- Without any neoclassical or $E \times B$ effects so that the residual momentum flux due to up-down flux-surface asymmetry can be quantified, $\Pi_{u d}$.

- With a background toroidal $E \times B$ flow gradient $\left(u^{\prime}\right)$ only so as to obtain a finite diffusive momentum flux and to calculate the toroidal viscosity coefficient, $\chi_{\phi, i}=\left(\Pi_{i}-\Pi_{u d}\right) / u^{\prime}$.

- With a finite bulk $E \times B$ rotation $(u)$ only so that the Coriolis pinch velocity, $V_{\phi}=\left(\Pi_{i}-\Pi_{u d} / u\right.$ can be calculated.

- With the finite $\rho_{*}$ corrections to the parallel derivatives [27] only, to calculate their contribution to the residual stress.

It is found that the predominant micro-instability in the core $\left(\rho_{T}=0.35\right)$ is the ion temperature gradient (ITG) instability. Figure 5 shows the mode frequency for the most unstable linear micro-instability for every point in the database for both radial points. Further out from the core $\left(\rho_{T}=0.5\right)$ the predominant instability becomes the Trapped Electron Mode (TEM) denoted by a negative (electron direction) mode frequency as the temperature, density gradients increase and the fraction of trapped particles becomes larger. In the bottom panel the quasi-linear electron-ion heat flux ratio is shown as a function of the mode frequency for both radial positions combined. We see the trapped electron modes having a larger electron heat flux with respect to the ion flux, however we do see some ITG modes where a larger electron heat flux is also seen (the opposite, trapped electron modes with predominant ion heat flux, is not seen).

Using Equation 1 a predicted toroidal $E \times B$ flow gradient, $u^{\prime}=-R^{2} / v_{t h i} \partial \Omega / \partial r$ can be calculated from the expression,

$$
u^{\prime}=-\frac{\Pi_{i}}{Q_{i}} \frac{R}{L_{T_{i}}} \frac{1}{P_{r}} \frac{v_{t h i}}{R} .
$$

by balancing the diffusive momentum flux with the residual stress (terms not proportional to $\mathrm{u}$ or $u^{\prime}$ and also encapsulating all the components related to 
Gyrokinetic investigation of intrinsic momentum in ASDEX Upgrade L-mode plasmas14
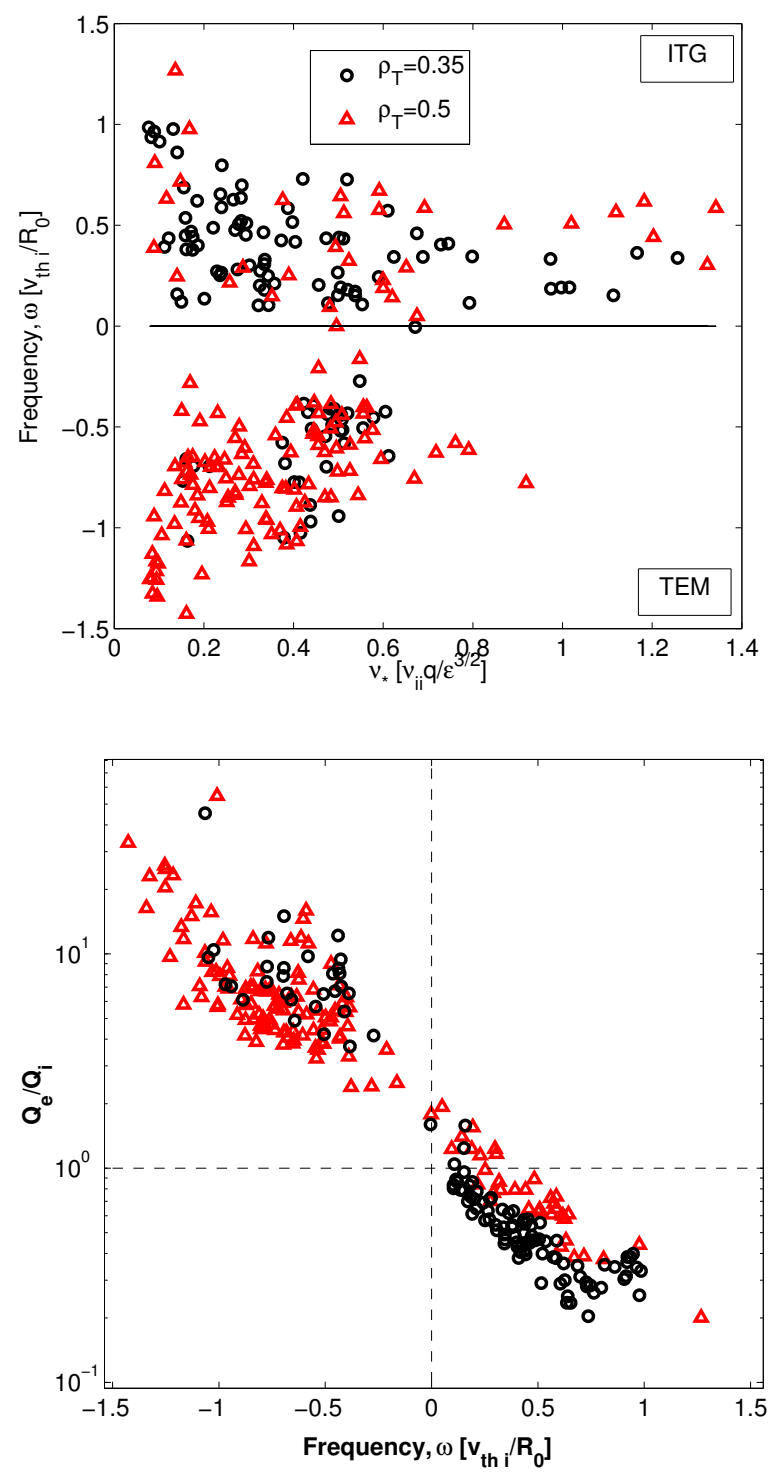

Figure 5. (top) The frequency of the most unstable mode for (black) $\rho_{T}=0.35$ and (red) $\rho_{T}=0.5$ as a function of the normalised ion-ion collision frequency, $\nu_{*}$. A positive frequency denotes a mode rotating in the ion-diamagnetic direction. (bottom) The ratio of the electron to ion radial quasi-linear heat fluxes as a function of the frequency of the most unstable mode. Vertical line denotes zero mode frequency and horizontal denotes a ratio of unity. 

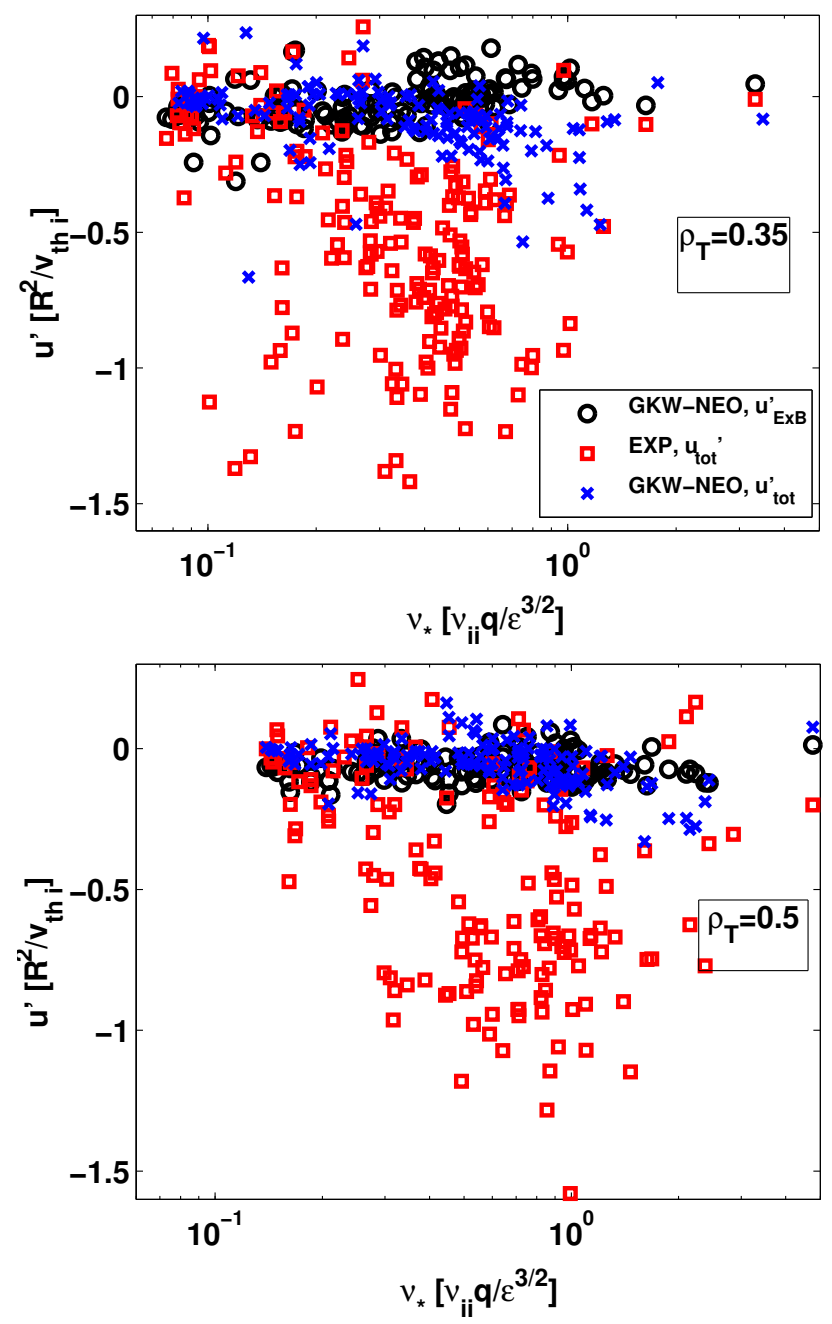

Figure 6. The radial gradient in the toroidal flow $(E \times B$ only (black circles) and total flow (blue crosses)) as predicted by quasi-linear gyrokinetic simulations and the value measured in the experiment (red squares) as a function of the normalised collision frequency for all the points in the L-mode database at the two radial points (top) $\rho_{T}=0.35$ and (bottom) $\rho_{T}=0.5$.

neoclassical flows and their radial gradients) [11]. This flow gradient is calculated using the ratio of the quasi-linear fluxes as calculated by GKW. The Prandtl number, $P_{r}$, defined as the ratio of the toroidal momentum and heat diffusivities, $P_{r}=\chi_{\phi, i} / \chi_{i}$. The quasi-linear Prandtl number is found to vary between, $0.45<P_{r}<2.0$ at $\rho_{T}=0.35$ and $0.69<P_{r}<2.5$ at $\rho_{T}=0.5$, consistent with previous studies, and experimentally measured values in other L-mode plasmas [61].

Fig. 6 shows the comparison of the flow gradients as calculated using Eq. 24 (black circles), against the experimentally measured value for both radial points (red circles). These are plotted as a function of the normalised ion-ion collisionality, $\nu_{i *}$. 

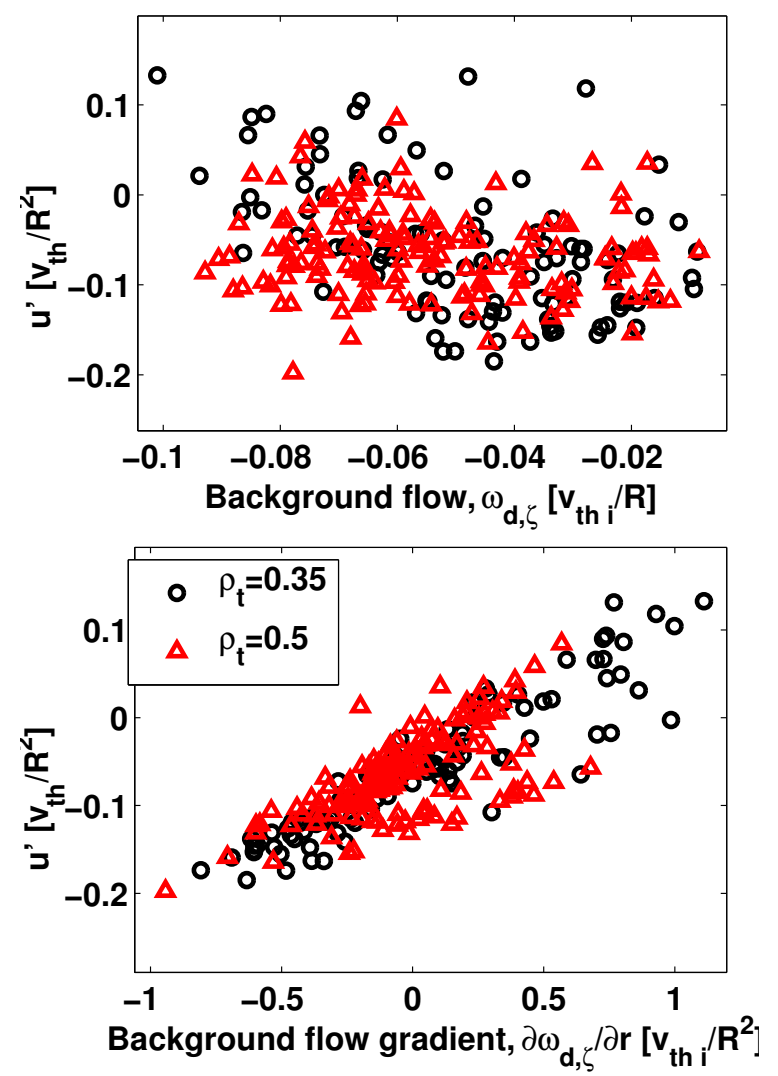

Figure 7. The $E \times B$ flow gradient maintained by neoclassical flows predicted by gyrokinetic simulations as a function of (top) the diamagnetic flow, and (bottom) radial gradient of the diamagnetic flow for all the points in the L-mode database.

The calculated values of $u^{\prime}$ vary across the database from $-0.35<u^{\prime}<0.13$ at $\rho_{t}=0.35$ and $-0.20<u^{\prime}<0.085$ at $\rho_{t}=0.5$. The experimental measurement is the total toroidal Boron flow, of which the $E \times B$ flow is a single component, as shown in Equation 2. Plotted also (blue crosses) is the total flow gradient $\left(u_{t o t}^{\prime}=u^{\prime}-\left(R_{0}^{2} / v_{t h i}\right) \partial u_{\text {neo }} / \partial r\right)$, taking the $E \times B$ flow as calculated by GKW and the diamagnetic and neoclassical components of the boron impurity flow as calculated by NEO and also including the effects of up-down asymmetry in the equilibrium.

A majority of points exhibit a hollow rotation profile $\left(u_{\text {tot }}^{\prime}<0\right)$, much like the experimentally measured values. However, in almost all cases the experimental measurements exhibit significantly larger gradients. Sometimes they are greater by a factor of 5-7 with a reasonable agreement seen only at low collisionality. Figure 7 shows the GKW-NEO computed values as a function of the neoclassical flows and their gradients. It is evident that there is a strong linear dependence of the sustained flow gradient with the background flow gradient, something which is not as evident against the flow amplitude, $\omega_{d, \zeta}$. This implies that the second derivatives of the density and temperature profiles are the critical parameters in such a model as these have a strong influence on the background flow gradient [38]. AUG has moderate collisionality with 


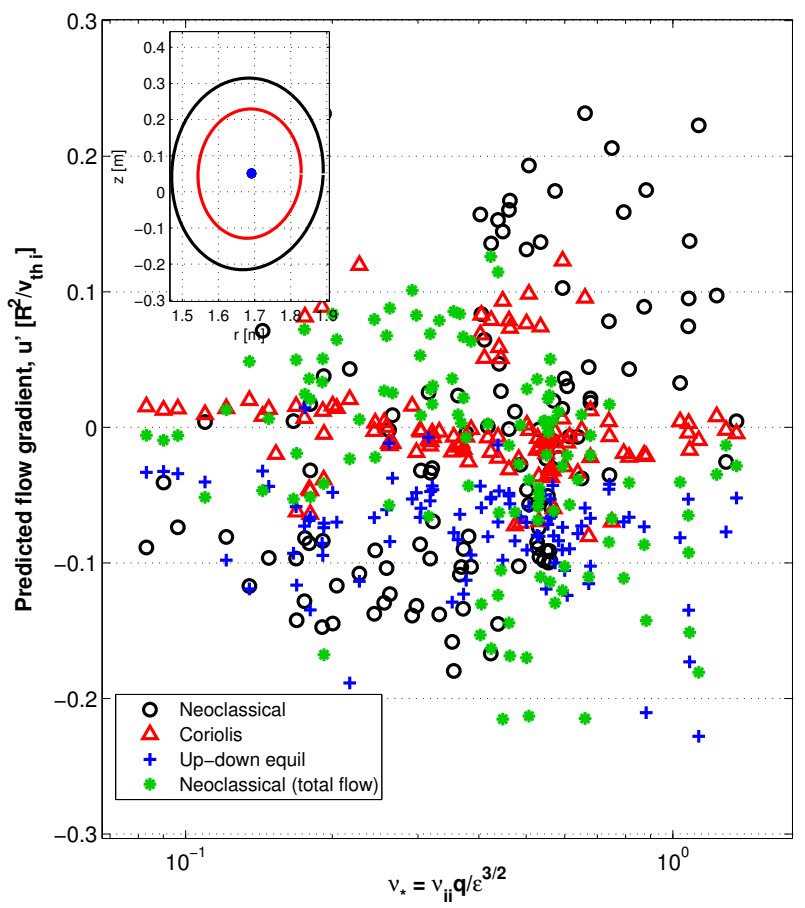

Figure 8. The flow gradient predicted by gyro-kinetic simulations as a function of collisionality for the different mechanisms considered at $\rho_{T}=0.35$. By neoclassical background flows (Black), momentum pinch (Red) and the up-down asymmetry in the equilibrium (Blue). The radial gradient in the total toroidal flow (green) when considering neoclassical effects is also shown. In inlay is an example of the flux-surfaces of AUG (2.8s, shot number 28243) showing the tilt, the (red) line is the $\rho_{T}=0.35$ flux surface while the (black) is the $\rho_{T}=0.5$. The (blue) dot is the magnetic axis.

a majority of the points sitting between $0.08<\nu_{*}<1.0$. In this range the ratio of $\Pi_{n e o, i} / Q_{i}$ due to neoclassical flows is seen to be weakly varying (See Fig 2).

In Figure 8, a comparison of the $E \times B$ flow gradients sustained by the three different mechanisms considered here is shown as well as the total flow gradient when considering a neoclassical background. Red triangles show the $u^{\prime}$ when only Coriolis drift effects are included. The gradient can be calculated by the expression, $u^{\prime}=-\left(R V_{\phi} / \chi_{i}\right)\left(u / P_{r}\right)$, the flow velocity used was the experimentally measured flow. It can be clearly seen that the Coriolis pinch is the smallest mechanism as the measured flows are small in these conditions of intrinsic rotation $(-0.07<u<0.07$ as seen in the bottom panel of Fig. 4). The black circles are those sustained by neoclassical flows. Here we subtract the value of the residual flux from up-down asymmetry, which is present in all simulations, so that a direct comparison between individual mechanisms can be made. A further residual stress contribution from finite $\rho_{*}$ corrections to the parallel derivatives were calculated. Their effects were seen to be very small, with a $u^{\prime}$ contribution of $u^{\prime} \sim 0.02$, which was independent of collisionality. It can be clearly seen from this analysis that the flow gradients sustained by neoclassical flows in AUG are of the same order as those produced by an up-down asymmetry of the flux surfaces. Blue 
crosses are from a separate simulation where no flows, flow gradients or neoclassical flows are considered and thus the only momentum flux generating mechanism is the residual stress due to up-down asymmetry (using the same expression as Eq. 24). The equilibrium flux surfaces have a finite elongation and tilt on AUG, an example is shown in inlay of Fig. 8 where the flux surface at $\rho_{T}=0.35$ is shown in (red) and $\rho_{T}=0.5$ in black.

This analysis indicates that the intrinsic rotation generated by neoclassical flows on their own is small by a factor as large as 5-7 compared to the toroidal rotation gradients measured on the AUG tokamak. Combining all the effects considered can sustain gradients that are slightly larger, $-0.6<u^{\prime}<0.2$, but these are also smaller in most cases than those measured experimentally. We proceed to analyse the sensitivity of these results to changes in plasma profiles.

\subsection{E×B perpendicular shear flow effects}

$\mathrm{E} \times \mathrm{B}$ shear flows have been shown to quench radial heat transport $[40,62,63,64,16]$, simultaneously they have been shown to induce radial anomalous toroidal momentum transport $[12,15,17,18,16]$. However, the sign of its contribution to the toroidal momentum flux may be positive or negative and as such can cause the effective Prandtl number to increase or decrease, depending on local parameters [15].

As such, the turbulent Prandtl number is a non-linear function of the flow shear rate. The $\mathrm{E} \times \mathrm{B}$ shear rate contribution from the toroidal velocity shear is defined as, $\gamma_{E}=\epsilon u^{\prime} / q$. In the previous section, the analysis was performed using a quasilinear calculation of the Prandtl number at the wave-number, $k_{\theta} \rho_{i}=0.42$. This did not include the effect of $\mathrm{E} \times \mathrm{B}$ shear flows. One way the quasi-linear analysis in the previous section would underestimate the sustained flow gradients is if the Prandtl number is overestimated significantly by the quasilinear calculation. An anomalously small effective turbulent Prandtl number, reduced by perpendicular flow shear would allow for significantly larger sustained gradients. Here the effective Prandtl number is defined (utilising the notation of [15]) by, $P_{r, \text { eff }}=\chi_{\phi \text {,eff }} / \chi_{i}$ where $\chi_{\phi, \text { eff }}=\chi_{\phi}+(\epsilon / q) M_{\|} . \quad M_{\|}$can be positive or negative depending on the sign of parallel and perpendicular flow gradients.

A selection of the database points were chosen at $\rho_{T}=0.35$ with a range of perpendicular flow shear rates, and a series of non-linear turbulence simulations were performed with and without imposed perpendicular shear flows (Using 41 toroidal modes, $k_{\theta} \rho_{i}^{\max }=2.0, \Delta k_{\theta} \rho_{i}=0.05,167$ radial modes,) and with parallel flow shear, $u^{\prime}$. The implementation of $\mathrm{E} \times \mathrm{B}$ perpendicular flow shear is described in [15, 40, 43]. Plotted in Fig. 9 are the turbulent Prandtl numbers as a function of the shear rate $\left(\gamma_{E}\right)$ consistent with the experimentally measured $u^{\prime}$. Across the whole database the shear rate varies between, $-0.12<\gamma_{E}<0.024$. We see that in general, and consistent with previous work (Fig. 14 from [15]), a 10-20\% reduction of the effective Prandtl number with perpendicular $\mathrm{E} \times \mathrm{B}$ flow shear is seen. All the cases we considered here have $\epsilon / q \sim 0.1$ and the magnetic shear, $\hat{s} \sim 0.5$.

The nonlinear Prandtl number here is also well approximated by the quasi-linear calculation (Horizontal dashed lines). It should be noted that the $u^{\prime}$ used here are the experimentally measured values, corrected for by removing the neoclassical background component, and not the quasi-linearly predicted flow gradients which are in almost all cases, smaller.

However, applying this observation to the AUG database, a small reduction due 
Gyrokinetic investigation of intrinsic momentum in ASDEX Upgrade L-mode plasmas19

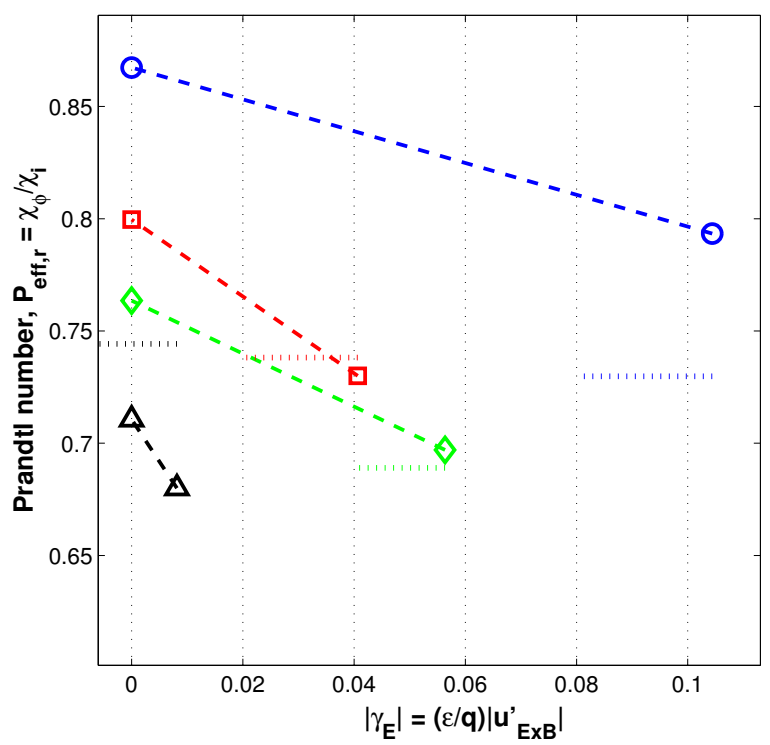

Figure 9. The effective turbulent Prandl number $\left(P_{r, \text { eff }}\right)$ with and without perpendicular $\mathrm{E} \times \mathrm{B}$ shear flows as calculated from separate non-linear turbulence simulations for four different database points (Parameters shown in Table 1) with the imposed flow shear rate, $\gamma_{E}$ calculated from the experimentally measured value of the $E \times B$ toroidal flow gradient, $u^{\prime}$ (dashed lines are plotted between the two corresponding simulations). Horizontal dashed lines are the corresponding Prandtl numbers as calculated from the quasi-linear analysis in the previous section and used to calculate the quasi-linear estimate of the flow gradients.

\begin{tabular}{l||c||c||c||c||r}
\hline label & $u_{\exp }^{\prime}$ & $u_{E \times B}^{\prime}$ & $\gamma_{E}$ & $\mathrm{q}$ & $\epsilon$ \\
\hline triangle & -0.07 & -0.083 & -0.0082 & 1.197 & 0.122 \\
square & -0.46 & -0.40 & -0.041 & 1.21 & 0.123 \\
diamond & -0.46 & -0.54 & -0.056 & 1.19 & 0.124 \\
circle & -0.71 & -1.01 & -0.104 & 1.20 & 0.124 \\
\hline
\end{tabular}

Table 1. Table showing shear parameters for the above nonlinear simulations. Showing the experiental flow gradient, purely $E \times B$ parallel flow gradient, perpendicular $E \times B$ shear rate, safety factor and inverse aspect ratio.

to $\mathrm{E} \times \mathrm{B}$ shear is not enough to account for the disparity in the predicted $u^{\prime}$ from our gyro-kinetic analysis to those experimentally measured which, in some cases, need a factor of 5-10 reduction in the effective Prandtl number for good agreement.

\subsection{Sensitivity to second derivatives of the density profile}

The sensitivity of the momentum flux on the neoclassical flow gradient (Fig. 7) suggests a test of whether, within the uncertainties of the experimental measurements, the disagreement between experimental results and predictions using nominal parameters shown in Fig. 6 can be significantly reduced.

Differently from ion and electron temperature, and toroidal rotation profiles, which are all based on local measurements with high radial resolution, the electron density profiles used in this study are based on the combination of line integrated 
signals (in the core) and local measurements (at the edge) by means of the integrated data analysis (IDA) technique [65]. Therefore uncertainties on the profile shapes of the core density profiles are large and it becomes important to verify whether, within the experimental error bars, predictions and measurements of the flow gradient can be reconciled. To this end, in this section we specifically consider the impact of the second derivatives of the density profiles and ask whether variations of the density profile are enough to explain the flow gradients observe and whether such perturbations of the density profile fall within the experimental error bars.

In section 2.4 we have discussed the dependence of the momentum flux driven by the radial gradient of the neoclassical flows and in turn on the radial second derivatives of the density and temperature. In Fig, 3, the dependence of the predicted momentum flux to ion heat flux ratio is a linear function of the second derivatives, where the slope of this linear dependence is function of various parameters, primarily the local $\rho_{*}$ value. By relying on this linear dependence to give a reasonable quasi-linear estimate, we can estimate the value of the second derivative of the main ion density profile which is required to obtain a prediction of the $E \times B u^{\prime}$ which matches the experimental value, obtained by subtracting the boron poloidal and diamagnetic components from the measured boron toroidal flow, according to Eq.2. The difference in the value of the second derivative can be related to the difference in flow gradient by the expression,

$$
\Delta \alpha=-\frac{2 P_{r} R / L_{n e}}{A R / L_{T i}} \Delta u_{E \times B}^{\prime}
$$

where $\alpha=-\left(R_{0}^{2} / n_{e}\right) \partial^{2} n_{e} / \partial r^{2}$ and $\mathrm{A}$ is the gradient of the fit which is scaled to the parameters of the individual experimental database point. The gradient is found to be independent of collisionality in the region of interest and linearly scales with the normalised ion-gyroradius $\left(\rho_{*}\right)$. For $\rho_{*}=0.002, A=0.006$.

The procedure is applied to all the points of the database and the required values of the second derivatives are compared to the nominal measured values in Fig. 10, at both $\rho_{T}=0.35$ (black) and $\rho_{T}=0.5$ (red), as a function of the measured $u^{\prime}$.

It is found that required values are up to one order of magnitude larger than the measured values, and that, particularly at $\rho_{T}=0.5$, they exhibit a clear trend with decreasing $u^{\prime}$. Moreover, the sign of the required second derivative is consistent with the nominal values at $\rho_{T}=0.35$, we observe that at 0.5 the required second derivative should have a positive values (in the definition of alpha this corresponds to concavity directed towards the bottom, whereas the majority of the profiles reconstructed from the measurements has opposite sign (concavity towards the top at $\rho_{T}=0.5$ ). An example of this difference is presented in lower panel of Fig.10. At the latter radial location, this is opposite in sign with respect to the second derivative which would be required to obtain the measured large negative values of $u^{\prime}$ at the same radial location, as shown in Fig. 10.

Local large increases of the second derivative of the profiles might still be considered to be compatible with the experimental measurements, particularly for the IDA reconstructed electron density profiles, the uncertainty in which are denoted by the grey dashed lines in Fig. 10. We note however that at large negative values of $u^{\prime}$, the required values of the second derivatives have the same sign at $\rho_{T}=0.35$ and $\rho_{T}=0.5$, which implies that consistency with the overall profile shape in that radial window would require an opposite and equally large second derivative in between those two radial locations, producing a compensating oscillation of the density profile shape in order to be consistent with the average first derivative of the profile over the same 
Gyrokinetic investigation of intrinsic momentum in ASDEX Upgrade L-mode plasmas 21
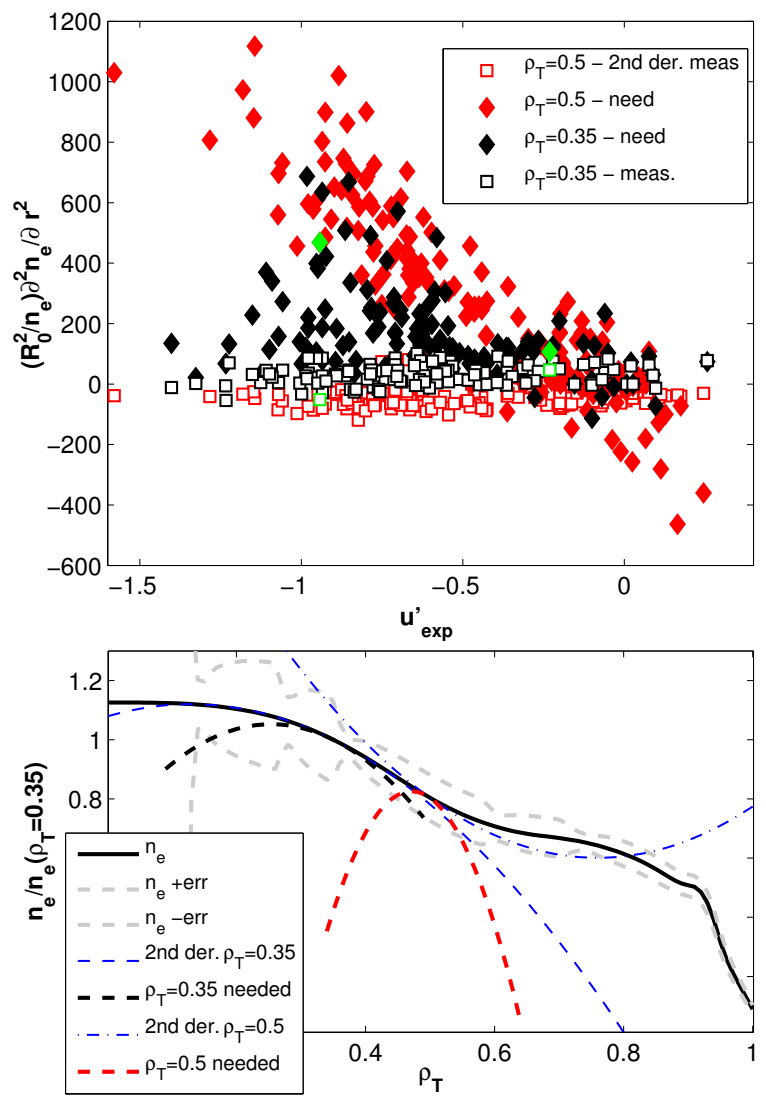

Figure 10. (Top) The required and measured second derivatives for all the points of the database against the experimentally measured flow gradient. (Green) points are the data points shown from the profile in the lower panel, where our analysis gave $u^{\prime}\left(\rho_{T}=0.35\right)=-0.26$ and $u^{\prime}\left(\rho_{T}=0.5\right)=0.0673$ respectively. (Bottom) Electron density profile, normalised to the $\rho_{T}=0.35$ surface (Black solid) and error bars (grey dashed) for shot \#28387, $t=3.1 \mathrm{~s}$. Plotted also are extrapolated profiles (from a second order Taylor expansion) at the $\rho_{T}=0.35$ (Blue dashed) and $\rho_{T}=0.5$ (Blue dot-dashed) surfaces used in our analysis. (Black) and (Red) dashed lines are examples of the local profiles with a local second derivative in the electron density needed, keeping the first derivative the same, to get agreement with the experimentally measured boron flow gradient.

radial window. The average first derivative has significantly smaller experimental uncertainties.

Measurements of the density profiles provided by the recently added ultra-fastswept reflectometer (UFSR) on AUG $[66,67]$ can be inspected to test whether these type of structures are present in the density profiles when moving from low to high density in $\mathrm{OH}$ plasmas. While uncertainties in the absolute position of the density measurements can be as large as $1 \mathrm{~cm}$ ( $2 \%$ of the minor radius), differences from two neighboring radial locations, as used in the calculation of a gradient, have much smaller uncertainties, so that the error bars in the calculation of first and second derivatives are certainly below $1 \%$. 
Gyrokinetic investigation of intrinsic momentum in ASDEX Upgrade L-mode plasmas22

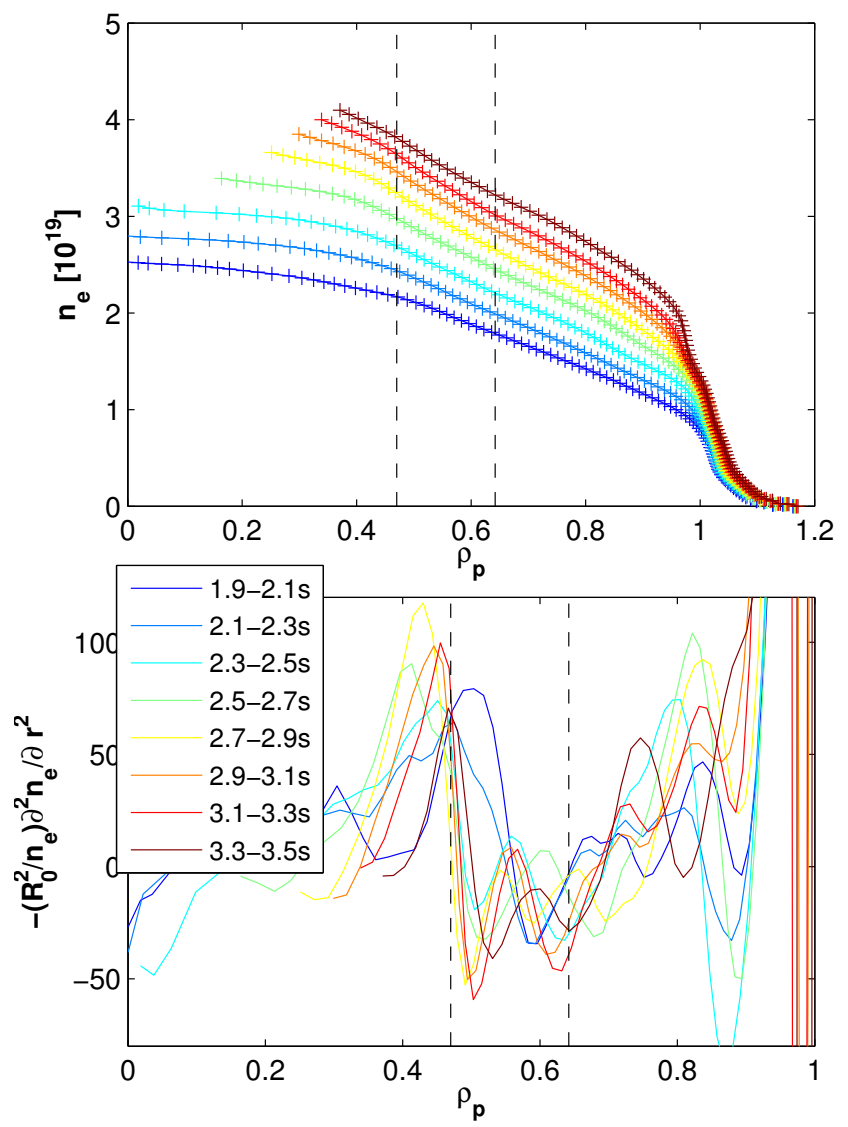

Figure 11. (Upper) Electron density profiles at different time slices from Shot \#32316 measured by the AUG ultra-fast-swept reflectometer. The profiles are time averaged in $0.2 \mathrm{~s}$ time windows. Error bars are not plotted as they are negligibly small. (Lower) The second derivative profiles of the electon density for the same time slices. The locations of our analysis are shown as dashed vertical lines.

The measured density profiles of a $\mathrm{OH}$ density ramp experiment at $1 \mathrm{MA}$ and $2.5 \mathrm{~T}$ (AUG discharge shot \#32316) are presented in Fig. 11, alongside with the computed profiles of the second derivatives. We observe that a characteristic structure is present in the profile shape in the radial window between $\rho_{p}=0.3$ and $\rho_{p}=0.6$, as shown in the lower panel of Fig. 11. However the size of the second derivatives extracted from these accurately measured profiles only ranges from -100 to +100 , roughly consistent with the IDA reconstructed density profiles, and exhibits an oscillation which is positive (concavity towards the bottom) at around $\rho_{p}=0.4$, and negative (concavity towards the top) around $\rho_{p}=0.5-0.6$.

In conclusion, the sensitivity of the results on the second derivatives of the profiles can be certainly used to decrease the disagreement between measured and predicted values of the flow gradient in several cases. However, this analysis provides clear evidence that that in some cases the second derivatives would have to be 4-10 
times larger than those obtained by fits to the experimental data to obtain a full compensation.

\section{Conclusions}

The effect of background neoclassical flows on turbulent momentum transport in the ASDEX Upgrade tokamak was studied. The interface between the neoclassical code NEO and the non-linear gyro-kinetic code GKW was implemented and benchmarked. This was then used to perform a quasi-linear analysis of turbulent intrinsic rotation on the ASDEX Upgrade Ohmic L-mode database. It should be noted that the quasilinear model used here does neglect some of the dependencies, for example the effects of $E \times B$ flows which are known to reduce the generated momentum flux and can change its magnitude and sign [29], which in turn could remove the need of the second derivative flipping sign.

In conclusion, the following was observed,

- The combined effects of neoclassical flows, $\mathrm{E} \times \mathrm{B}$ velocity flow shear, Coriolis pinch and up-down asymmetry in the equilibrium is insufficient to explain the flow gradients that are measured, which are greater by factor of between 3-5 but in some cases by an order of magnitude.

- The flow gradients sustained by the momentum flux generated by neoclassical flows are of the same order as those generated by the Coriolis pinch and up-down equilibrium asymmetry.

- Non-linear simulations calculating the turbulent Prandtl number, and its reduction due to $E \times B$ flow shear, showed that its effect is small and the reduction not enough to give a quantitative agreement between this analysis and the experimental profiles but did show that the quasi-linearly calculated values of the fluxes and Prandtl numbers closely approximate the fully non-linearly calculated values.

- The flow gradients are directly proportional to the local second derivative of the density and temperature gradients. The residual momentum flux is strongly related to the flow gradient. Using an order of magnitude estimate based on our quasilinear simulation results, we have matched the second derivatives at the $\rho_{T}=0.35$ and $\rho_{T}=0.5$ surfaces to give agreement between our analysis and the experimental profiles. The required local second derivatives would have to be 4-10 times larger than those obtained by fits to the experimental data to obtain agreement which would require local profiles to have corrugations whose amplitudes are inconsistent with measured profiles.

- The neoclassical flow induced momentum fluxes obtained in non-linear turbulence simulations are seen to be very similar to the quasi-linear value. In fact, the quasilinear values slightly overestimate the full non-linear calculation, and as such the predicted $u^{\prime}$ values can be slightly overestimated.

A wide variety of momentum transport mechanisms have been suggested in the literature. Here they have been compared systematically and directly with a database of 186 measured profiles from AUG. Gyrokinetic calculations have been performed at nominal parameters and the gradients of intrinsic rotation generated by the combined effects of neoclassical flows, up-down asymmetry and the Coriolis pinch are significantly smaller than the maximum gradients observed. Some of the 
Gyrokinetic investigation of intrinsic momentum in ASDEX Upgrade L-mode plasmas 24

further known symmetry breaking effects, such as profile shearing and other nonlinear mechansims can contribute significantly to the measured intrinsic rotation. These require a global description of the plasma turbulence and will be investigated in a future publication.

\section{Appendix A}

For the sake of completeness and to document exactly which equations are being solved, the fully normalised source terms are shown here. The notation and normalisations used are given in Ref. [43], and the reader is referred to this paper for further details. We not that the wave-vectors are always normalised to the ion gyro-radius $\rho_{i}, k_{\psi} \rightarrow k_{\psi} \rho_{i}$. Subscript $\mathrm{N}$ denotes a normalised quantity and the Roman numerals label the terms as denoted in Ref. [27].

The equation can be written in the form

$$
\frac{\partial f}{\partial t}=\mathrm{III}+\mathrm{IV}+\mathrm{V}+\mathrm{VII}+\mathrm{VIII} .
$$

Only terms that are modified by the neoclassical background are described here, then each term is, in its normalised form:

$$
\begin{aligned}
& \mathrm{III} \rightarrow-\mathbf{v}_{E} \cdot \nabla f=\frac{\mathbf{b} \times \nabla \phi}{B} \cdot \nabla f \rightarrow \\
& \imath k_{\zeta} \varepsilon \zeta \frac{\partial \phi_{\text {neo }}}{\partial \psi} f+\imath k_{\zeta} \varepsilon^{s \zeta} \frac{\partial \phi_{\text {neo }}}{\partial s} f+\imath k_{\psi} \varepsilon^{s \psi} \frac{\partial \phi_{\text {neo }}}{\partial s} f \\
& \mathrm{IV} \rightarrow-\frac{1}{m v_{\|}}\left(v_{\|} \mathbf{b} \cdot(\mu \nabla B+Z e \nabla \phi)\right) \frac{\partial f}{\partial v_{\|}} \\
& =-\frac{1}{m}\left(\mathbf{b} \cdot\left(\mu \nabla B+Z e \nabla \phi_{\text {neo }}\right)\right) \frac{\partial f}{\partial v_{\|}} \\
& \rightarrow-\left(\mu_{N} v_{s} \mathcal{F} \frac{\partial B_{N}}{\partial s}+\frac{v_{s} Z}{2 T_{s}} \mathcal{F} \frac{\partial \phi_{\text {neo }, \mathrm{N}}}{\partial s}\right) \frac{\partial f}{\partial v_{\| N}} \\
& \mathrm{~V} \rightarrow-\mathbf{v}_{E} \cdot \nabla F_{0}=\frac{\mathbf{b} \times \nabla x^{\alpha}}{B} \imath k_{\alpha} \phi \cdot \nabla F_{0} \rightarrow \\
& \varepsilon^{\alpha \psi} \imath \rho_{i} k_{\alpha} \cdot \nabla \psi_{N} \frac{\partial F_{M}}{\partial \psi} \phi_{N}+\imath \varepsilon^{\alpha \beta} \rho_{i} k_{\alpha} \phi_{N} \frac{\partial F_{\text {neo }}}{\partial x^{\beta}} \\
& \mathrm{VII} \rightarrow \frac{Z e}{m_{s}} \mathbf{b} \cdot \nabla \phi \frac{\partial F}{\partial v_{\|}} \rightarrow \\
& -\frac{Z v_{s}}{T_{s}} v_{\| N} \mathcal{F} \frac{\partial \phi_{N}}{\partial s} F_{M}+\frac{Z v_{s}}{2 T_{s}} \mathcal{F} \frac{\partial \phi_{N}}{\partial s} \frac{\partial F_{\text {neo }}}{\partial v_{N \|}} \\
& \mathrm{VIII} \rightarrow-\frac{Z e}{v_{\|} m_{s}} \overrightarrow{v_{D}} \cdot \nabla \phi \frac{\partial F}{\partial v_{\|}} \rightarrow
\end{aligned}
$$


Gyrokinetic investigation of intrinsic momentum in ASDEX Upgrade L-mode plasmas 25

$$
-i \frac{k_{\alpha} Z}{T_{s}} \overrightarrow{v_{D}} \cdot \nabla x^{\alpha} \phi_{N} F_{M}+\frac{\imath k_{\alpha} Z}{2 T_{s} v_{\| N}} \overrightarrow{v_{D}} \cdot \nabla x_{a} \phi_{N} \frac{\partial F_{\text {neo }}}{\partial v_{\| N}}
$$

\section{Acknowledgments}

A part of this work was carried out using the HELIOS supercomputer system at Computational Simulation Centre of International Fusion Energy Research Centre (IFERC-CSC), Aomori, Japan, under the Broader Approach collaboration between Euratom and Japan, implemented by Fusion for Energy and JAEA.

The authors would like to thank E. Belli for making the NEO code available and for their help. Many useful discussions with F.J. Casson and Y. Camenen are also acknowledged. Many thanks to A. Medvedeva for supplying the reflectometry measurements from ASDEX Upgrade.

\section{References}

[1] J.E. Rice et al 2007 Nucl. Fusion 471618

[2] H. Biglari, P. H. Diamond, and P. W. Terry, 1990 Phys. Fluids B 2, 1

[3] R. E. Waltz, G. D. Kerbel, J. Milovich, and G. W. Hammett, 1995 Phys. Plasmas 2, 2408

[4] G. M. Waltz, R. Staebler, R. E. Waltz, J. Candy, and J. E. Kinsey,2013 Phys. Rev. Lett. 110, 055003

[5] P.A. Politzer et al 2008 Nucl. Fusion 48, 075001

[6] H. Reimerdes et al 2007 Phys. Rev. Lett. 98, 055001

[7] A. G. Peeters et al 2005, Phys. Plasmas 12, 072515

[8] F.I. Parra et al 2011 Phys. Plasmas 18, 062501

[9] A.G. Peeters et al 2011 Nucl. Fusion 51094027

[10] F. Parra and M Barnes, 2015, Plasma Phys. Control. Fusion 57, 045002

[11] P.H. Diamond et al 2009 Nucl. Fusion 49045002

[12] R.R. Dominguez and G.M. Staebler, 1993 Phys. Fluids B 5, 3876

[13] X. Garbet et al 2002, Phys. Plasmas 9, 3893

[14] Ö. D. Gürcan et al 2007 Phys. Plasmas 14042306

[15] F.J. Casson et al 2009, Phys. Plasmas 16, 092303

[16] G. Staebler et al 2013 Phys. Rev. Lett. 110055003

[17] R.E. Waltz et al Phys. Plasmas 14, 122507 (2007)

[18] M. Barnes et al 2011 Phys. Rev. Lett. 106175004

[19] A. G. Peeters, C. Angioni and D. Strintzi 2007 Phys. Rev. Lett. 98265003

[20] T.S. Hahm et al 2007 Phys. Plasmas 14072302

[21] A. G. Peeters et al 2009, Phys. Plasmas 16, 042310

[22] N. Kluy et al 2009 Phys. Plasmas 16122302

[23] H. Weisen et al 2012 Nucl. Fusion 52042001

[24] Y. Camenen et al 2009, Phys. Plasmas 16, 012503

[25] Y. Camenen et al 2009, Phys. Rev. Lett. 102, 125001

[26] J Ball et al 2016 Plasma Phys. Control. Fusion 58045023

[27] T. Sung et al 2013, Phys. Plasmas 20, 042506

[28] F. I. Parra et al, (2011) Nucl. Fusion 51113001

[29] M. Barnes et al 2013 Phys. Rev. Lett. 111055005

[30] R. Buchholz et al 2014 Phys. Plasmas 21062304

[31] Y. Camenen et al 2011 Nucl. Fusion 51073039

[32] Z.X. Lu 2015 Phys. Plasmas 052118

[33] Z.X. Lu et al 2015 Nucl. Fusion 55093012

[34] Ö. D. Gürcan et al 2010 Phys. Plasmas 17112309

[35] F.L. Hinton and R.D. Hazeltine Rev. Mod. Phys. 48239 (1976)

[36] J. Lee et al 2014 Nuclear Fusion 54022002

[37] J. Lee et al., 2014 Phys Plasmas 21056106

[38] J. Lee et al 2015 Plasma Phys. Control. Fusion 57125006

[39] S. P. Hirshman and D. J. Sigmar (1981) Nucl. Fusion 21, 1079 
Gyrokinetic investigation of intrinsic momentum in ASDEX Upgrade L-mode plasmas 26

[40] Y. Camenen et al, (2016) Phys. Plasmas 23, 022507

[41] J.D. Callen et al (2009) Nucl. Fusion 49085021

[42] R.M. McDermott et al 2014 Nucl. Fusion 54043009

[43] A.G. Peeters et al 2009, Computer Physics Communications 1802650

[44] E A Belli and J Candy 2009 Plasma Phys. Control. Fusion 51075018

[45] E A Belli and J Candy 2012 Plasma Phys. Control. Fusion 54015015

[46] E A Belli and J Candy 2008 Plasma Phys. Control. Fusion 50095010

[47] F.J. Casson et al 2010, Phys. Plasmas 17, 102305

[48] S. Hamada, Kakuyugo Kenkyu 1, 542 (1958)

[49] C.F.F. Karney, Comp. Phys. Reports 4 (1986) 183

[50] W. Dorland et al., (2000) Phys. Rev. Lett. 85, 5579

[51] A.M. Dimits, Phys. Plasmas 7 (2000) 969

[52] X. Lapillonne et al 2009 Phys. Plasmas 16032308

[53] M.A. Beer et al 1995 Phys. Plasmas 2, 2687

[54] P. Manas To be submitted.

[55] Y.B. Kim, P.H. Diamond and R.J. Groebner, (1991) Phys. Fluids B 3, 2050

[56] J .C. Hillesheim et al 2015 Nucl. Fusion 55032003

[57] F. Parra Private Communication (2015)

[58] C. Angioni et al 2011 Phys. Rev. Lett. 107215003

[59] R M McDermott et al 2011 Plasma Phys. Control. Fusion 53124013

[60] H.Lütjens, A. Bondeson and O. Sauter, Computer Physics Communications 97 219-260 (1996)

[61] T. Tala et al 2011 Nucl. Fusion 51123002

[62] K.H. Burrell 1997 Phys. Plasmas 41499

[63] J.E. Kinsey, R.E. Waltz and J.Candy 2005 Phys. Plasmas 12062302

[64] J.E. Kinsey, R.E. Waltz and J.Candy 2007 Phys. Plasmas 14102306

[65] R. Fischer et al 2010 Fusion Science and Technology 58, 2

[66] F. Clairet et al., Rev. Sci. Instrum 201081 10D903.

[67] A. Medvedeva et al., Density fluctuations measurements with an ultra-fast-swept reflectometer in ASDEX Upgrade. (2015) In 12th International Reflectometry Workshop (IRW 12) 